\title{
Intermixture of positive and negative trials in instrumental discrimination ${ }^{1}$
}

\author{
JOHN W. DAVENPORT AND WILLIAM W. HAGQUIST \\ REGIONAL PRIMATE RESEARCH CENTER, UNIVERSITY OF WISCONSIN
}

\begin{abstract}
The incompleteness of conventional discrimination theories is indicated by the finding that, under some conditions, rats do not develop an instrumental discrimination when normallysufficient numbers of positive and negative trials are given in unmixed fashion ( $60 \mathrm{~S}+$ trials followed by $60 \mathrm{~S}$-trials) instead of the customary random intermixture of $S+$ and $S$ - trials.
\end{abstract}

It is standard practice in animal studies of instrumental (nonchoice) discrimination to schedule positive (S+) and negative (S-) trials in intermixed fashion, e.g., ++-+--+-+-. Such scheduling may be regarded as being near the end of a continuous dimension which is definable in terms of the number of positive-to-negative and negative-to-positive transitions in a schedule and which can range from single alternation, through intermixed blocks of consecutive S+ or S- trials, to the completely unmixed condition in which all positive trials precede all negative trials. In this report we present some preliminary data from an automated runway situation with rats on the question of whether discrimination is impaired or facilitated when the degree of intermixture is considerably lower than normal.

Method

The Ss were 24 male Holtzman albino rats which were 135 days old and experimentally naive at the start of training.

Two automatic $Y$ mazes, enclosed in plywood boxes and controlled remotely by standard operant conditioning equipment, were used as runways. The left arm of each $Y$ maze was partitioned off to form a doorless runway consisting of a startbox (9.5 in. long), an alley (28 in. long), and a goal area (4 in. long). The height of each area was 5 in.; widths varied from 3.5 in. to 7 in. The apparatuses were painted flat black and were equipped with food cups at the rear of the startbox and at the end of each arm, 1 in. green cue lights over each food cup, $45 \mathrm{mg}$ pellet dispensers, photocells adjacent to the food cups and at the point of exit from the startbox, and a Clare metal-enclosed relay behind the choice point of the $Y$ maze which provided a clicking sound when energized by a $10 / \mathrm{sec}$ stream of pulses.

After magazine training (60 pellets in $30 \mathrm{~min}$ ), discrete locomotion trials began in pretraining on Days 1 and 2. On these days the Ss were trained (20 trials/S) to initiate trials by interrupting the photobeam at the rear of the startbox in the presence of the startbox light. A trial was defined by the duration of the goalarea light. Trial initiation was rewarded by a single pellet in the startbox on each of the pretraining trials but was not rewarded thereafter. Completion of the trial (turning around in the startbox and traversing the alley) was rewarded by a single pellet in the goal area. Between trials, Ss were in darkness but free to move in any portion of the runway. Throughout the experiment, intertrial intervals were determined by a variableinterval 30 sec tape program and the fact that, with both apparatuses in use simultaneously, one $S$ received trials during another's intertrial intervals and could not initiate a trial until after the other S's trial was completed; this resulted in actual intertrial intervals ranging from $20 \mathrm{sec}$ to $2 \mathrm{~min}$.

On Days 3-14, 120 training trials were given at the rate of 10/day. For each S, 60 of these were rewarded (1 pellet, produced by interruption of the goal-area photobeam, with a $5 \mathrm{sec}$ continuation of the trial stimulus following pellet delivery), and 60 were nonrewarded (5 sec light continuation only). The rewarded and nonrewarded trials were distinguished by the presence or absence of the click, which had the same onset and offset as the trial stimulus when presented.

The Ss were randomly assigned to six treatment combinations of a factorial design in which there were three degrees of trial intermixture (Randomized Trials, Randomized Blocks, or Unmixed) and two cue-reward relationships (Click Positive vs Click Negative). The Randomized Trials (RT) subgroups received their S+ and $S-$ training trials in the customary intermixed fashion within each session (sequences varied over days) with the restriction of no more than two consecutive S+ or S- trials. The Randomized Blocks (RB) subgroups received intermixed blocks of five consecutive $\mathrm{S}+$ or five consecutive S- trials, with the order of blocks the same as the order of individual trials in the RT condition. The Unmixed (U) subgroups received $60 \mathrm{~S}+$ trials (first six days of the training phase) followed by $60 \mathrm{~S}$ - trials (last six days).

Following the training phase, a 110 trial test phase was given over Days 15-25 in which all Ss received differential conditioning trials in the customary intermixed manner, i.e., as in the RT condition of the training phase, with the restriction that the first trial of the first test session (Day 15) was always an S+ trial. The relationship between cues (click vs no click) and and reward conditions (+ vs -) in the test phase remained the same as in the training phase for each $\mathrm{S}$.

Running speed (100/time) over a 32 in. distance from the startbox to the goal area was taken on all trials. Resulis

Figure 1 presents the running speed data for the six subgroups over the entire course of the experiment, 


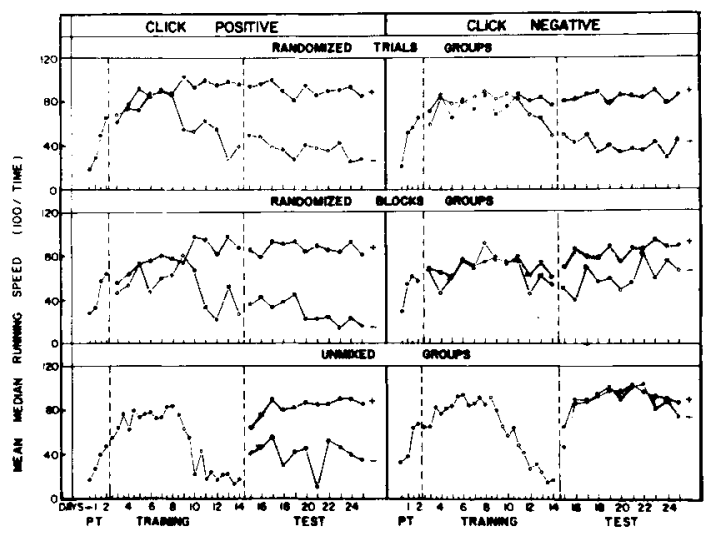

Fig. 1. Running-speed curves for the six subgroups over the pretraining (PT), training, and test phases.

with discrimination (superiority of S+ over S- speed) indicated by shading. This figure shows that degree of intermixture in the training phase produced, at best, only a small effect upon discriminative performance in the test phase in the Click Positive subgroups but had a very pronounced effect in the Click Negative subgroups. The Unmixed Click Negative subgroup showed no discrimination over most of the test phase, and showed little or no savings in comparison to the training phase of the RT Click Negative subgroup, after displaying fairly complete extinction to S- in the training phase.

In an analysis of variance of the test phase data, the triple interaction of schedules, cues, and rewardnonreward which is suggested in Fig. 1 was insignificant $(F=1.39$, $d f=2 / 12)$; but the interaction between schedules and cues attained significance $(F=5.25, \mathrm{df}=$ $2 / 12, p<.05$ ), and the interaction between schedules and reward-nonreward approached significance $(F=2.93$, $\mathrm{df}=2 / 12, \mathrm{p}<.10$ ). A general tendency for faster running to be elicited by the click stimulus was reflected in a significant main effect of cues $(F=12.15$, df $=1 / 12$, $\mathrm{p}<.01)$, a significant interaction between cues and reward-nonreward $(F=11.39$, df $=1 / 12, p<.01)$, and, as may be seen in Fig. 1, subgroup differences during the training phase.

Six of the eight Unmixed Ss, including all four in the Click Negative subgroup, failed to show any indication of discrimination throughout the first test-phase session (Day 15). With the exception of one $S$ in the RB Click Negative subgroup, all other Ss showed clear discrimination from the outset of this session.

\section{Diseussion}

The experiment indicated that there are some conditions under which the formation of an instrumental discrimination may not take place unless S+ and Strials are intermixed to some degree. Hopefully, investigators working with other discrimination apparatuses (e.g., black-white runways) and in other discrimination paradigms (e.g., differential classical conditioning) will provide data which will shed light on the generality of this nondiscrimination effect.

Conventional theories of discrimination learning have not explicitly predicted differing performance levels for varying degrees of trial intermixture. If this variable proves to be a factor of wide generality, theoretical accounts of discrimination which claim to be complete will have to specify what facilitative process occurs as $\mathrm{S}$ goes from trial to trial in an intermixed sequence of S+ and S- trials to produce better discrimination than that obtained in unmixed conditions. At this stage it seems appropriate to confine our suggestions to the bare mention of such possibilities as attentional factors, differences between the intermixed and unmixed conditions in the frustrative value of nonrewarded trials (or reinforcing value of rewarded trials), stimulus aftereffects persisting between trials, or some perceptual process which is dependent on the frequency of trialto-trial stimulus transitions.

\section{Note}

1. This study was supported by Grant FR-00167 from the National Institutes of Health. 